\title{
A ranking model for the greedy algorithm and discrete convexity
}

\author{
Ulrich Faigle • Walter Kern • Britta Peis
}

Received: 6 July 2009 / Accepted: 21 April 2010 / Published online: 4 September 2010

(C) The Author(s) 2010. This article is published with open access at Springerlink.com

\begin{abstract}
Generalizing the idea of the Lovász extension of a set function and the discrete Choquet integral, we introduce a combinatorial model that allows us to define and analyze matroid-type greedy algorithms. The model is based on a real-valued function $v$ on a (finite) family of sets which yields the constraints of a combinatorial linear program. Moreover, $v$ gives rise to a ranking and selection procedure for the elements of the ground set $N$ and thus implies a greedy algorithm for the linear program. It is proved that the greedy algorithm is guaranteed to produce primal and dual optimal solutions if and only if an associated functional on $\mathbb{R}^{N}$ is concave. Previous matroid-type greedy models are shown to fit into the present general context. In particular, a general model for combinatorial optimization under supermodular constraints is presented which guarantees the greedy algorithm to work.
\end{abstract}

Mathematics Subject Classification (2000) 90C27 · 90C57

\section{Introduction}

The "greedy algorithm" represents one of the most fundamental (but not necessarily optimal) algorithmic principles: always take the locally best step! The question arises when optimality of the greedy solution can be guaranteed. A well-known and

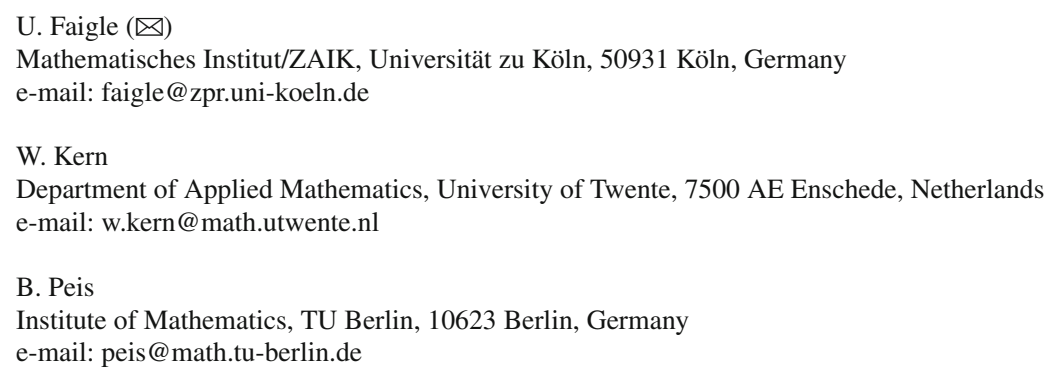


important model for the latter are matroids (in particular, in Edmonds' [5] polyhedral model), where the greedy algorithm successfully solves certain combinatorial linear programs (see also, e.g., Fujishige [14], Faigle and Fujishige [7]). The greedy principle shows up in even wider contexts (see, e.g., Hoffman $[17,19])$ and appears to be generally closely connected with the algorithmic theory of combinatorial optimization (cf. Hoffman and Schwartz $[16,18]$ ).

There have been many generalizations of the matroid-greedy model. Frank's [11] supermodular model is one of the farthest-reaching and derives the greedy algorithm from a 2-phase procedure, where a pair of dual linear programs is solved (thus generalizing the approach of Faigle and Kern [9]). Often, however, "the greedy algorithm" is viewed (and analyzed) as a one-sided procedure with respect to just one in the pairs of linear programs (see, e.g., the greedy algorithm of Dietrich and Hoffman [3] or Fujishige's [13] dual greedy algorithm).

The main contribution of the present investigation is a model that comprises and extends all of the aforementioned polyhedral matroid-type greedy models. Moreover, our model offers an integrated approach with respect to the underlying primal-dual linear programs. We arrive at a greedy algorithm through a notion of ranking selections, based on the restriction function in the combinatorial model. We then show that the greedy algorithm is guaranteed to be successful for one type of linear program if and only if it solves the other type as well (Theorem 5.1). Generalizing the approach of Fujishige [13] and the Monge extensions of the game-theoretic model of Faigle et al. [8], our model associates with the greedy algorithm a certain functional, which turns out to be concave if and only if the greedy algorithm works. This greedy functional generalizes the so-called Lovász extension of a set function (which coincides with Choquet's [2] discrete integral).

Lovász [21] observed in the set function context that the concavity of the greedy functional is equivalent to the supermodularity of the underlying restriction function. This observation has initiated a theory of "discrete convexity" that investigates subor supermodular type functions on the lattice of integer points in $\mathbb{R}^{n}$ ( $c f$. Murota [22]). Note that our theory differs substantially from the latter. Concavity (or convexity, when seen from a maximizing point of view) of the greedy functional does not rely on notions of super- or submodularity and hence suggests a new model for "discrete convexity".

Nevertheless, traditional models for successful matroid-type greedy algorithms all involve a notion of sub- and supermodularity. We present a general model for supermodularity in Sect. 6 and prove supermodularity of the restriction function to be sufficient for the greedy algorithm to work. Curiously, a similarly general model seems to be much harder to establish with respect to optimization under submodular constraints. So we do not take it into consideration here. (For some results on optimization under generalized submodular constraints, see, e.g., Faigle and Peis [10].)

\section{Combinatorial linear programs}

Let $N$ be a finite set and $\mathcal{F}$ a family of non-empty subsets of $N$. A valuation is a function $v: \mathcal{F} \rightarrow \mathbb{R}$ (resp. $v \in \mathbb{R}^{\mathcal{F}}$ ). It is convenient to extend $\mathcal{F}$ to $\mathcal{F}_{0}=\mathcal{F} \cup\{\emptyset\}$ 
and to set $v(\emptyset)=0$. Moreover, we use the notation

$$
\mathcal{F}(X)=\{F \in \mathcal{F} \mid F \subseteq X\} \text { for any } X \subseteq N
$$

Given a valuation $v$ and a parameter vector $c \in \mathbb{R}^{N}$, we refer to the optimization problem

$$
\min \langle c, x\rangle \text { s.t. } \quad x_{i} \geq 0 \forall i \in N \quad \text { and } \quad x(F) \geq v(F) \forall F \in \mathcal{F}
$$

as a combinatorial linear program. As usual, $\langle c, x\rangle=\sum_{i \in N} c_{i} x_{i}$ denotes the inner product of $c$ and $x$ and $x(F)=\left\langle\mathbf{1}^{F}, x\right\rangle$, where $\mathbf{1}^{F} \in\{0,1\}^{N}$ is the incidence vector of the subset $F$ with

$$
\mathbf{1}_{i}^{F}=1 \Longleftrightarrow i \in F
$$

Remark In certain specific contexts, $x \mapsto\langle c, x\rangle$ is often called the cost function of the optimization problem (1).

The linear programming dual of (1) is:

$$
\max \langle v, y\rangle \text { s.t. } y_{F} \geq 0 \forall F \in \mathcal{F} \text { and } \sum_{F \ni i} y_{F} \leq c_{i} \forall i \in N \text {. }
$$

Defining the non-negative function $v^{+}: \mathcal{F}_{0} \rightarrow \mathbb{R}_{+}$by

$$
v^{+}(F)=\max \{v(F), 0\} \quad(F \in \mathcal{F}),
$$

we may replace $v$ by $v^{+}$without affecting the feasibility region of (1):

$$
\mathbb{P}_{+}(v)=\left\{x \in \mathbb{R}_{+}^{N} \mid x(F) \geq v(F) \forall F \in \mathcal{F}\right\}=\mathbb{P}_{+}\left(v^{+}\right)
$$

Observe that (1) is bounded if and only if $c \in \mathbb{R}_{+}^{N}$. Hence we will usually assume that $c$ and $v$ are non-negative. In this case, both linear programs are feasible and hence have optimal solutions.

\section{Greedy rankings}

We take a greedy approach to the linear programming problems (1) and (2). Our algorithm consists of two phases. In the first phase, we construct a feasible solution $y$ for the linear program (2) in a greedy fashion. At the same time, we choose a sequence $\pi$ of representatives for the sets in $\mathcal{F}$ by selecting members of $N$ into $\pi$ that yield tight constraints during the construction of $y$. The second phase of the algorithm will construct a candidate solution $h^{\pi}$ from $\pi$ for the linear program (1). 


\subsection{Ranking functions}

A ranking function for $(\mathcal{F}, v)$ is an operator $X \mapsto \mu(X)$ on the subsets of $N$ such that for all $X \subseteq N$,

$$
\mu(X) \in \mathcal{F}_{0}(X) \text { and } v(\mu(X))=\max _{F \in \mathcal{F}_{0}(X)} v(F)
$$

Example 3.1 The greedy algorithm of Hoffman [17] arranges $\mathcal{F}$ in a linear order $\mathcal{F}=\left(F_{1}, \ldots, F_{m}\right)$ according to non-increasing $v$-values and works with the ranking function $\mu_{H}(X)=F_{i}$, where $F_{i} \in \mathcal{F}(X)$ has the smallest possible index $i$. (See also Sect. 6 below.)

Remark A ranking function is a special case of a choice function in the sense of Moulin [23]. Choice functions underly also Fujishige's [13] model for dual greedy algorithms.

\subsection{The greedy algorithm}

Given the ranking function $\mu$, we execute the following procedure for any non-negative input parameter vector $c \in \mathbb{R}_{+}^{N}$ and select a sequence $\pi=p_{1} \ldots p_{k}$ of elements of $N$ as representatives of the members of $\mathcal{F}$ so that each $p_{i}$ is chosen from the currently ranked set. As usual, we denote the empty string by $\pi=\square$.

\section{Greedy Algorithm}

$\left(\mathrm{G}_{0}\right)$ Initialize: $X \leftarrow N, \pi \leftarrow \square, \gamma \leftarrow c, y \leftarrow 0$,

$\left(\mathrm{G}_{1}\right)$ WhILE $\mu(X) \neq \emptyset$ DO:

SELECT some $p \in \mu(X)$ of minimal current weight $\gamma_{p}$;

UPDATE $y_{\mu(X)} \leftarrow \gamma_{p}, \pi \leftarrow[\pi p]$;

UPDATE $\gamma_{q} \leftarrow\left[\gamma_{q}-\gamma_{p}\right]$ for all $q \in \mu(X), X \leftarrow[X \backslash p]$;

The procedure returns a sequence $\pi=p_{1} \ldots p_{k}$ of elements of $N$ (with possibly $k<|N|$ ) and a non-negative parameter vector $y \in \mathbb{R}^{\mathcal{F}}$, to which we refer as the greedy solution. The sequence $\pi$ is called a $\mu$-ranking of $N$ (or just a ranking, for short). So $\pi=p_{1} \ldots p_{k}$ is a $\mu$-ranking if and only if $p_{i} \in \mu\left(N \backslash\left\{p_{1}, \ldots, p_{k}\right\}\right)$ and $\mu\left(N \backslash\left\{p_{1}, \ldots, p_{k}\right\}\right)=\emptyset$.

The family $\mathcal{M}$ of sets $\mu(X)$ considered in the course of the construction of the ranking $\pi$ is the family of Monge sets of $\pi$. So with the notation $X_{1}=N$ and $X_{i+1}=X_{i} \backslash p_{i}$ for $i=1, \ldots, k$, we have $M_{k+1}=\mu\left(X_{k+1}\right)=\emptyset$ and

$$
\mathcal{M}=\left\{M_{1}=\mu\left(X_{1}\right), \quad M_{2}=\mu\left(X_{2}\right), \ldots, M_{k}=\mu\left(X_{k}\right)\right\},
$$

$p_{i} \in M_{i} \backslash M_{i+1}$ for all $i=1, \ldots, k$. Note that $\mathcal{M}$ is uniquely determined by the ranking $\pi$.

Lemma 3.1 Let $(\pi, y)$ be the output of the greedy algorithm relative to the input $c \geq 0$. Then the following holds: 
(0) $y$ is non-negative.

(1) $\sum_{F \ni p} y_{F} \leq c_{p}$ for all $p \in N$.

(2) $\sum_{F \ni p} y_{F}=c_{p}$ for all $p$ in $\pi$.

In particular, y is a feasible solution for (2).

Proof The properties (0-2) are immediate consequences of the selection and update rules in step $\left(\mathrm{G}_{1}\right)$ of the algorithm.

We denote by $\Pi=\Pi(\mathcal{F}, v, \mu)$ the collection of all rankings for all $c \geq 0$.

Remark The type of algorithm considered in this section is also called "dual greedy" ( $c f$. Fujishige [13]) as it works on the "dual" optimization problem (2). We prefer the term "greedy" because the algorithm constructs a ranking $\pi$ greedily with respect to the $\mathcal{F}$-valuation $v$.

\section{Greedy functionals and kernels}

The selection rule $\left(\mathrm{G}_{1}\right)$ of the greedy algorithm leaves some freedom in the choice of a representative $p \in \mu(X)$ of minimal current weight. This does not affect the greedy solution $y$, however.

Lemma 4.1 The greedy solution $y$ is uniquely determined by the input $c \geq 0$ into the greedy ranking algorithm.

Proof Suppose, for example, that $p \in \mu(N)$ was selected in step $\left(\mathrm{G}_{1}\right)$, while $q \in \mu(N)$ would have been a candidate as well. Let $M$ be the first subsequent choice of a Monge set with a non-trivial value $y_{M}>0$. In view of $y_{\mu(N)}=c_{p}=c_{q}$, it is then clear that $M \in \mathcal{F}(N \backslash\{p, q\})$ must hold. Hence the same $y$ would have resulted from the choice $q \in \mu(N)$ as a representative.

We may thus associate with the ranking structure $(\mathcal{F}, v, \mu)$ a well-defined functional $v_{\mu}: \mathbb{R}^{N} \rightarrow \mathbb{R} \cup\{-\infty\}$ via

$$
v_{\mu}(c)=\left\{\begin{array}{cl}
\langle v, y\rangle & \text { if } c \geq 0 \\
-\infty & \text { otherwise }
\end{array}\right.
$$

where $y=y(c)$ is the greedy solution with respect to $c$, and define the $\mu$-kernel of $(\mathcal{F}, v)$ as the closed convex set

$$
\operatorname{ker}_{\mu}(v)=\left\{x \in \mathbb{R}^{N} \mid\langle c, x\rangle \geq v_{\mu}(c) \forall c \in \mathbb{R}^{N}\right\}
$$

Proposition 4.1 $\operatorname{ker}_{\mu}(v)=\mathbb{P}_{+}(v)$. 
Proof Because $v_{\mu}(c)$ is non-negative for every $c \geq 0$, we conclude $x \geq 0$ for every $x \in \operatorname{ker}_{\mu}(v)$. Letting $\mathbf{1}_{F} \in\{0,1\}^{N}$ be the incidence vector of $F$, the greedy algorithm yields $v_{\mu}\left(\mathbf{1}_{F}\right) \geq v(F)$ for any $F \in \mathcal{F}$ and, therefore, $\operatorname{ker}_{\mu}(v) \subseteq \mathbb{P}_{+}(v)$.

Conversely, any $z \in \mathbb{P}_{+}(v)$ is a feasible solution for the linear program (1), while $y$ is feasible for $(2)$. So $\mathbb{P}_{+}(v) \subseteq \operatorname{ker}_{\mu}(v)$ follows from linear programming duality in view of

$$
\langle c, z\rangle \geq\langle y, v\rangle=v_{\mu}(c) \text { for all } c \in \mathbb{R}_{+}^{N} .
$$

Note that the greedy functional $v_{\mu}$ is positively homogeneous in the sense

$$
v_{\mu}(\lambda v)=\lambda v_{\mu}(c) \text { for all } \lambda>0 .
$$

Recall that $v_{\mu}$ is said to be concave if for all $c, d \in \mathbb{R}^{N}$ and $0 \leq t \leq 1$,

$$
v_{\mu}(t c+(1-t) d) \geq t v_{\mu}(c)+(1-t) v_{\mu}(d) .
$$

It follows that the greedy algorithm is guaranteed to be optimal for the dual linear program (2) if and only if the associated greedy functional is concave:

Theorem 4.1 The following statements are equivalent:

1. $v_{\mu}: \mathbb{R}^{N} \rightarrow \mathbb{R} \cup\{-\infty\}$ is concave;

2. $v_{\mu}(c)=\min \left\{\langle c, x\rangle \mid x \in \mathbb{P}_{+}(v)\right\} \quad$ for all $c \in \mathbb{R}_{+}^{N}$.

3. $v_{\mu}(c)=\max \left\{\langle v, y\rangle \mid y \geq 0, \sum_{F \ni i} y_{F} \leq c_{i} \quad \forall i \in N\right\}$ for all $c \in \mathbb{R}_{+}^{N}$.

Proof In view of Proposition 4.1, the equivalence of the first two statements comes from a well-known fact in convex analysis ( $c f$. Rockafellar [24]). The last two statements are equivalent by linear programming duality.

Example 4.1 In the case of $\mathcal{F}_{0}=2^{N}$ and of a non-decreasing set function $v: \mathcal{F} \rightarrow \mathbb{R}$, the ranking function $\mu(X)=X$ yields $v_{\mu}(c)$ as the value of the so-called Lovász extension of $v$ for every $c \geq 0$ ( $c f$. Lovász [21]).

\section{Marginal vectors and convexity}

While Theorem 4.1 characterizes combinatorial structures $(\mathcal{F}, v)$ for which the greedy algorithm solves the dual linear program (2) optimally, we now turn our attention to the primal linear program (1).

\subsection{Marginal vectors}

Given a ranking $\pi=p_{1} \ldots p_{k}$ with associated family $\mathcal{M}$ of Monge sets returned by the greedy algorithm, a straightforward candidate solution for the linear program (1) 
exists. Observe that $\pi$ and $\mathcal{M}$ induce a unique vector $h^{\pi} \in \mathbb{R}^{N}$ with the property

$$
\begin{array}{ll}
\text { (i) } h_{p}^{\pi}=0 & \text { if } p \text { does not occur in } \pi \\
\text { (ii) } h^{\pi}(M)=v(M) & \text { for all } M \in \mathcal{M} .
\end{array}
$$

Indeed, the $(\pi \times \mathcal{M})$-incidence matrix $\mathbb{A}=\left[a_{i j}\right] \in \mathbb{R}^{k \times k}$ with coefficients

$$
a_{i j}= \begin{cases}1 & \text { if } p_{i} \in M_{j} \\ 0 & \text { otherwise }\end{cases}
$$

is (lower) triangular with diagonal elements $a_{i i}=1$ and hence invertible and therefore yields a unique solution for the equations (i) and (ii). We call $h^{\pi}$ the marginal vector of $(\mathcal{F}, v)$ relative to the ranking $\pi$. Observe that this notion coincides with the classical definition of marginal vectors

$$
h^{\pi}\left(p_{i}\right)=v\left(\left\{p_{i}, \ldots, p_{n}\right\}\right)-v\left(\left\{p_{i-1}, \ldots, p_{n}\right\}\right)
$$

when $\mathcal{F}_{0}$ comprises all subsets of $N$.

Lemma 5.1 Let $(\pi, y)$ be the output of the greedy algorithm for $c \geq 0$. Then one has

$$
\left\langle c, h^{\pi}\right\rangle=\sum_{p \in \pi} c_{p} h_{p}^{\pi}=\sum_{M \in \mathcal{M}} v(M) y_{M}=\langle v, y\rangle=v_{\mu}(c) .
$$

Proof Let $\tilde{h}$ be the restriction of $h^{\pi}$ to $\pi$ and $\tilde{v}$ the restriction of $v$ to the Monge set $\mathcal{M}$ of $\pi$. Then we have $\tilde{h}^{T} \mathbb{A}=\tilde{v}^{T}$ from property (ii) of a marginal vector.

On the other hand, property (2) of Lemma 3.1 above says $\mathbb{A} \tilde{y}=\tilde{c}$, where we have restricted $\tilde{y}$ similarly to $\mathcal{M}$. Hence we deduce:

$$
\sum_{p \in N} c_{p} h_{p}^{\pi}=\langle\tilde{c}, \tilde{h}\rangle=\langle\tilde{h}, \tilde{c}\rangle=\left\langle\tilde{v}, \mathbb{A}^{-1} \tilde{c}\right\rangle=\langle\tilde{v}, \tilde{y}\rangle=\sum_{F \in \mathcal{F}} v(F) y_{F} .
$$

\subsection{Convexity}

We now characterize the structures where the marginal vectors $h^{\pi}$ yield optimal solutions for the linear program $(1)$. We say that $(\mathcal{F}, v, \mu)$ is convex if each marginal vector $h^{\pi}$ lies in $\mathbb{P}_{+}(v)$, i.e., if each $h^{\pi}$ is a feasible solution for (1). (See Sect. 6 for some generic examples of convex structures.)

Theorem 5.1 The following statements are equivalent:

(a) $(\mathcal{F}, v, \mu)$ is convex.

(b) For every $c \in \mathbb{R}_{+}^{N}$, the corresponding marginal vector $h^{\pi}$ is optimal for the linear program (1). 
(c) For every $c \in \mathbb{R}_{+}^{N}$, the corresponding greedy solution $y$ is optimal for the linear program (2).

(d) The greedy functional $v_{\mu}: \mathbb{R}^{N} \rightarrow \mathbb{R} \cup\{-\infty\}$ is concave.

Proof We first show (a) $\Rightarrow$ (b), (c). Assume that $(\mathcal{F}, v, \mu)$ is convex and let $y$ be the greedy solution relative to $c \geq 0$. Then $h^{\pi}$ is feasible for (1) while $y$ is feasible for (2). In view of $\left\langle c, h^{\pi}\right\rangle=\langle v, y\rangle$ (Lemma 5.1), the optimality of $h^{\pi}$ and of $y$ follows from linear programming duality.

For $(\mathrm{c}) \Rightarrow$ (a), let $\pi=p_{1} \ldots p_{k}$ be some ranking with associated Monge sets $M_{1}, \ldots, M_{k}$ and marginal vector $h^{\pi}$. By Lemma 5.2 (below), we may assume that $\pi$ arises from the greedy algorithm relative to some $c \geq 0$ such that the greedy vector $y$ satisfies:

(i) $y_{M_{s}}>0$ for all $s=1, \ldots, k$.

(ii) $\sum_{F \ni p} y_{F}<c_{p}$ for each $p \notin \pi$.

Let $x^{*}$ be an optimal solution for the linear program (1). Since $y$ is optimal for (2), the complementary slackness conditions hold. So we conclude:

$$
\begin{aligned}
& y_{F}>0 \Longrightarrow x^{*}(F)=v(F) . \\
& x_{p}^{*}>0 \Longrightarrow \sum_{F \ni p} y_{F}=c_{p} .
\end{aligned}
$$

In view of (ii), the latter conditions imply $x_{p}^{*}=0$ for all $p \notin \pi$. So (i) says that $x^{*}$ satisfies the defining conditions of $h^{\pi}$, which yields $h^{\pi}=x^{*} \in \mathbb{P}_{+}(v)$. The equivalence of (c) and (d) was exhibited in Theorem 4.1.

Remark Given Theorem 5.1, one might want to call convex structures $(\mathcal{F}, v, \mu)$ rather 'concave'. With the present terminology, we follow the terminology of cooperative game theory that calls a game 'convex' if all marginal vectors lie in $\mathbb{P}_{+}(v)$ (cf. Shapley [25]).

Lemma 5.2 Let $\pi=p_{1} p_{2} \ldots p_{k}$ be an arbitrary ranking for $(\mathcal{F}, v)$ with Monge family $\mathcal{M}=\left\{M_{1}, M_{2}, \ldots, M_{k}\right\}$. Then there exists a parameter vector $\tilde{c}$ with positive components $\tilde{c}_{p}>0$ such that the greedy algorithm outputs the pair $(\pi, \tilde{y})$ with the properties

(i) $\tilde{y}_{M_{s}}>0$ for all $s=1, \ldots, k$.

(ii) $\sum_{F \ni p} \tilde{y}_{F}<\tilde{c}_{p}$ for each $p \notin \pi$.

Proof Arguing by induction, let $N^{\prime}=N \backslash\left\{p_{1}\right\}$ and assume that the ranking $\pi^{\prime}=$ $p_{2} \ldots p_{k}$ and the greedy vector $y \in \mathbb{R}_{+}^{\mathcal{F}\left(N^{\prime}\right)}$ are produced by the greedy algorithm relative to some positive $c \in \mathbb{R}^{N^{\prime}}$ such that

(i) $y_{M_{s}}>0$ for all $s=2, \ldots, k$.

(ii) $\sum_{F \ni p} y_{F}<c_{p}$ for each $p \notin \pi$. 
Note that $\mathcal{M}^{\prime}=\left\{M_{2}, \ldots, M_{k}\right\}$ is the Monge family of $\pi^{\prime}$ and construct $\tilde{c}$ from $c$ as follows:

$$
\tilde{c}_{p}= \begin{cases}1 & \text { if } p=p_{1} \\ 1+c_{p} & \text { if } p \in M_{1} \backslash\left\{p_{1}\right\} \\ c_{p} & \text { otherwise }\end{cases}
$$

Relative to $\tilde{c}$, the greedy algorithm will produce the ranking $\pi=p_{1} p_{2} \ldots p_{k}$ and the greedy solution $\tilde{y}$ with the property

$$
\tilde{y}_{F}= \begin{cases}1 & \text { for } F=M_{1} \\ y_{F} & \text { for } F \in\left\{M_{2}, \ldots, M_{k}\right\} \\ 0 & \text { otherwise, }\end{cases}
$$

which proves the Lemma.

\section{Examples}

We exhibit some generic convex combinatorial structures. An important feature of these structures is the existence of a consecutive (partial) precedence ordering $(\mathcal{F}, \preceq$ ). Convexity is guaranteed if the valuation $v: \mathcal{F} \rightarrow \mathbb{R}$ is sufficiently order-compatible (i.e., in a sense monotone and supermodular). Frank's model for a greedy algorithm arises as a special case in this framework. Furthermore, Dijkstra's shortest path algorithm, Edmond's algorithm for minimal rooted branchings, and Ford and Fulkerson's maximum flow algorithm for planar graphs can be viewed as further examples.

\subsection{Consecutive orders}

A (partial) ordering $(\mathcal{F}, \preceq)$ is called consecutive if for all $F^{\prime}, F, F^{\prime \prime} \in \mathcal{F}$,

(C) $F^{\prime} \preceq F \preceq F^{\prime \prime} \Longrightarrow F^{\prime} \cap F^{\prime \prime} \subseteq F$.

We interpret $F^{\prime} \preceq F$ as a dominance relation, where $F^{\prime}$ is "preferred" to $F$. Consecutive dominance orders always exist. For example, the trivial order on $\mathcal{F}$ (with no comparable pairs of elements) is consecutive. Also the set-theoretic containment order $(\mathcal{F}, \subseteq$ ) is consecutive (and yields the combinatorial model investigated in [8]). Often more refined consecutive orderings are "natural".

Example 6.1 (Cuts) Let $\mathcal{C}$ consist of the non-empty cut sets

$$
\delta^{+}(S)=\{(x, y) \in A \mid x \in S, y \notin S\}
$$

of the digraph $G=(V, A)$. Then $(\mathcal{C}, \preceq)$ is consecutive, where

$$
\delta^{+}(S) \preceq \delta^{+}(T) \quad \Longleftrightarrow \quad S \supseteq T
$$


Example 6.2 (Planar flows) Let $G=(V, E)$ be a (directed or undirected) graph in a planar representation with specified vertices $s, t$ (i.e., $G$ is a so-called $(s, t)$-planar graph). There exists an intuitive partial order " $\preceq$ " on the collection $\mathcal{P}$ of all cycle-free $(s, t)$-paths: For any $P, Q \in \mathcal{P}$, we define

$$
P \preceq Q \quad \Longleftrightarrow \quad P \text { "lies below" } Q
$$

and find that $(P, \preceq)$ is consecutive.

Example 6.3 (Convex geometries and posets) Let $X \mapsto \bar{X}$ be a closure operator with ground set $N$ such that every $S \subseteq N$ admits a unique minimal subset $\operatorname{ext}(S) \subseteq S$ of so-called extreme points with $S \subseteq \overline{\operatorname{ext}(S)}$. (The associated closure space is a convex geometry in the sense of Edelman and Jamison [6]). Order the family $\mathcal{E}=\{\operatorname{ext}(S) \mid$ $S \subseteq N\}$ via

$$
\operatorname{ext}(S) \preceq \operatorname{ext}(T) \Longleftrightarrow \bar{S} \supseteq \bar{T}
$$

Then $(\mathcal{E}, \preceq)$ is consecutive. A well-studied special class of such structures arises from partial orders $P=(N, \leq)$, when $\mathcal{E}$ is taken as the collection of all antichains of $P$. The trivial closure $\bar{X}=X$ yields $\mathcal{E}$ as the collection of all subsets of $N$.

In the following, we will always assume that $(\mathcal{F}, \preceq)$ is consecutive and the valuation $v: \mathcal{F} \rightarrow \mathbb{R}_{+}$is monotone in the sense

$$
F^{\prime} \preceq F^{\prime \prime} \quad \Longrightarrow \quad v\left(F^{\prime}\right) \geq v\left(F^{\prime \prime}\right) \quad\left(F^{\prime}, F^{\prime \prime} \in \mathcal{F}\right) .
$$

Hence we may assume $\mathcal{F}=\left\{F_{1}, \ldots, F_{m}\right\}$ to be arranged such that

(i) $F_{i} \prec F_{j} \Longrightarrow i<j$.

(ii) $v\left(F_{1}\right) \geq \cdots \geq v\left(F_{m}\right)$.

\subsection{Sub- and supermodularity}

A submodular pair relative to $(\mathcal{F}, \preceq)$ is a pair $(F, G)$ of distinct sets $F, G \in \mathcal{F}$ that admits sets $F \vee G, F \wedge G \in \mathcal{F}(F \cup G)$ with the property

$$
F \wedge G \preceq F, G \preceq F \vee G
$$

For example, $(F, G)$ is a submodular pair when $F \prec G$ holds: It suffices to set $F \vee G=F$ and $F \wedge G=G$. The terminology is motivated by the following observation:

Lemma 6.1 Let $(\mathcal{F}, \preceq)$ be consecutive and $\chi_{p}: \mathcal{F} \rightarrow\{0,1\}$ the indicator function of $p \in N$. Then the submodular inequality holds for every submodular pair $(F, G)$ :

$$
\chi_{p}(F \wedge G)+\chi_{p}(F \vee G) \leq \chi_{p}(F)+\chi_{p}(G)
$$


Remark The indicator function may be replaced by any non-negative vector in Lemma 6.1.

A submodular family for $(\mathcal{F}, \preceq)$ is a collection $\mathcal{S}$ of submodular pairs $(F, G)$ that includes all comparable pairs, i.e., for all $F, G \in \mathcal{F}$,

$$
F \prec G \quad \Longrightarrow \quad(F, G) \in \mathcal{S} \text {. }
$$

We refer to $(F, G) \in \mathcal{S}$ as an $\mathcal{S}$-pair.

Example 6.4 Let $(\mathcal{E}, \preceq)$ be the ordered family of extremal sets of a convex geometry (cf. Ex. 6.3). Let $\mathcal{S}$ be the collection of all pairs $(F, G)$ of distinct sets $F, G \in \mathcal{E}$. Then $\mathcal{S}$ is a submodular family in view of the operations

$$
F \wedge G=\operatorname{ext}(F \cup P) \text { and } F \vee G=\operatorname{ext}(F \cap G)
$$

We say that the valuation $v: \mathcal{F} \rightarrow \mathbb{R}_{+}$is $\mathcal{S}$-monotone if for every $F \in \mathcal{F}$ and collection $\mathcal{G} \subseteq \mathcal{F}$ of sets with $F \preceq G$ for all $G \in \mathcal{G}$ the following is true:

$(\mathrm{SM})$ either $v(F) \geq \sum_{G \in \mathcal{G}} v(G)$ or $\mathcal{G}$ contains an $\mathcal{S}$-pair.

The singleton case $\mathcal{G}=\{G\}$ exhibits an $\mathcal{S}$-monotone valuation to be monotone in particular.

Example 6.5 Assume that $\mathcal{S}$ is a submodular family such that

(D) $\left(G, G^{\prime}\right) \in \mathcal{S}$ is guaranteed for the distinct sets $G, G^{\prime}$ if some jointly dominating $F \in \mathcal{F}$ with $F \preceq G, G^{\prime}$ exists.

Then every monotone valuation is trivially $\mathcal{S}$-monotone. (Not only Ex. 6.4 has property (D). Also the more general model of Frank [11] enjoys (D).)

Relative to a given submodular family $\mathcal{S}$, we say that $v$ is $\mathcal{S}$-supermodular if the following inequality holds for every $(F, G) \in \mathcal{S}$ :

$$
v(F \wedge G)+v(F \vee G) \geq v(F)+v(G)
$$

Note that the supermodular inequality holds trivially in the case $F \prec G$. Hence it suffices to investigate it on incomparable $\mathcal{S}$-pairs.

\subsection{Supermodularity in intersecting systems}

A submodular family $\mathcal{S}$ is said to be intersecting (relative to $(\mathcal{F}, \preceq$ ) if for all incomparable sets $F, G \in \mathcal{F}$,

(I) $F \cap G \neq \emptyset \Longrightarrow(F, G) \in \mathcal{S}$.

Our next result generalizes Frank's [11] greedy algorithm. 
Theorem 6.1 Let $(\mathcal{F}, \preceq)$ be consecutive and $\mathcal{S}$ an intersecting and submodular family of pairs. Let $v: \mathcal{F} \rightarrow \mathbb{R}_{+}$be an $\mathcal{S}$-monotone and $\mathcal{S}$-supermodular valuation. Then $(\mathcal{F}, v, \mu)$ is convex for every ranking function $\mu$ such that $\mu(X)$ is always a maximal member of $(\mathcal{F}(X), \preceq)$.

Proof Let $\pi=p_{1}, \ldots, p_{k}$ be the ranking and $\mathcal{M}=\left\{M_{1}, \ldots, M_{k}\right\}$ be the family of Monge sets relative to the input $c \geq 0$ and ranking function $\mu$. We must show that the greedy algorithm provides an optimal solution for the linear program

$$
\max _{y \geq 0} \sum_{F \in \mathcal{F}} v(F) y_{F} \quad \text { s.t. } \quad \sum_{F \ni p} y_{F} \leq c_{p} \forall p \in N
$$

To this end, we order $\mathcal{F}_{0}=\left(F_{1}, \ldots, F_{m}\right)$ linearly so that $F_{1}=M_{1}=\mu(N)$ and for all $F_{i}, F_{j}$,

$$
F_{i} \preceq F_{j} \Longrightarrow i \leq j .
$$

Let $\bar{y}$ be the greedy solution and $y^{*}$ the lexicographically maximal optimal solution with respect to the fixed order on $\mathcal{F}_{0}$. Arguing by induction, we will show $\bar{y}=y^{*}$.

If $\bar{y}_{F_{1}}=y_{F_{1}}^{*}$, we apply the induction hypothesis to $\mathcal{F}\left(N \backslash p_{1}\right)$ and find that $y^{*}$ and $\bar{y}$, in fact, agree on all components. So it suffices to demonstrate that $\bar{y}_{F_{1}}>y_{F_{1}}^{*}$ would contradict the choice of $y^{*}$ as the lexicographically maximal optimal solution.

Note first that the $\operatorname{support} \operatorname{supp}\left(y^{*}\right)=\left\{F \in \mathcal{F} \mid y_{F}^{*}>0\right\}$ of $y^{*}$ cannot contain any incomparable $\mathcal{S}$-pair $(F, G)$. Adding some $\varepsilon>0$ to the components $y_{F \wedge G}^{*}$ and $y_{F \vee G}^{*}$ and subtracting $\varepsilon$ from the components $y_{F}^{*}$ and $y_{G}^{*}$ would otherwise produce a lexicographically larger vector, which is feasible by the submodularity of $\mathcal{S}$ (Lemma 6.1) and still optimal by the supermodularity of $v$.

Consider the family $\mathcal{G} \subseteq \operatorname{supp}\left(y^{*}\right)$ of immediate successors of $F_{1}$ in the support of $y^{*}$ (relative to the order relation $\preceq$ ). Since $\mathcal{S}$ is intersecting, any two intersecting sets $F, G \in \operatorname{supp}\left(y^{*}\right)$ must be comparable. Hence the consecutiveness of $(\mathcal{F}, \preceq)$ implies that every element $p \in F_{1}$ that could stop us from increasing $y_{F_{1}}^{*}$ towards $\bar{y}_{F_{1}}$ feasibly must occur in some set $G \in \mathcal{G}$. So we obtain another feasible (and lexicographically larger!) solution $\tilde{y}$ from $y^{*}$ when we increase $y_{F_{1}}^{*}$ by some $\varepsilon>0$ and simultaneously decrease $y_{G}^{*}$ for all $G \in \mathcal{G}$ by the same amount $\varepsilon$.

On the other hand, the sets in $\mathcal{G}$ are pairwise incomparable. So $\mathcal{G}$ contains no $\mathcal{S}$-pair at all. Hence the $\mathcal{S}$-monotonicity of $v$ yields

$$
v\left(F_{1}\right) \geq \sum_{G \in \mathcal{G}} v(G)
$$

Therefore, $\tilde{y}$ would also be optimal and thus contradict the choice of $y^{*}$.

6.4 Cuts, arborescenses and planar flows

We give two illustrations of Theorem 6.1. 


\subsubsection{Cuts and arborescences}

Let $G=(V, A)$ be a directed graph with a fixed root $s \in V$ such that any $t \in V$ can be reached from $s$ by some directed path. Consider the family of $s$-cuts

$$
\mathcal{C}_{s}=\left\{\delta^{+}(S) \mid s \in S, S \neq V\right\}
$$

with the order $\left(\mathcal{C}_{S}, \preceq\right)$ as in Ex. 6.1. Define for any $S, T \subseteq V$ with $s \in S \cap T$ and $S \cup T \neq V$,

$$
\begin{aligned}
& \delta^{+}(S) \wedge \delta^{+}(T)=\delta^{+}(S \cup T) \\
& \delta^{+}(S) \vee \delta^{+}(T)=\delta^{+}(S \cap T) .
\end{aligned}
$$

The family $\mathcal{S}$ of all incomparable pairs of cuts is submodular. Moreover, the valuation $v$ with $v(\emptyset)=0$ and $v(C)=1$ otherwise is trivially $\mathcal{S}$-monotone and supermodular. Frank [11] has observed that $\left(\mathcal{C}_{s}, \preceq\right)$ is an intersecting system as in Ex. 6.5 and that the associated pair of optimization problems of type (1) and (2) can be solved greedily.

Indeed, Theorem 6.1 guarantees $\left(\mathcal{C}_{s}, \preceq\right)$ to be convex. Consequently, the greedy algorithm produces a ranking $\pi$ so that the associated marginal vector $h^{\pi}$ has $(0,1)$ components and satisfies

$$
h^{\pi}\left(\delta^{+}(S)\right) \geq 1 \quad(s \in S, S \neq V) .
$$

This marginal vector is the incidence vector of an s-branching, i.e., an arc set that admits directed paths from $s$ to any $t \in V$. If $A$ is weighted with nonnegative weights $c_{a} \geq 0$, the greedy algorithm will produce an $s$-branching of minimal weight, which solves the problem considered by Fulkerson [15]. For general weights $c_{a}$, the greedy algorithm will produce an $s$-branching which solves Fulkerson's problem (including Edmonds' [4] $s$-branchings and min-cost spanning trees as special cases).

If furthermore some $t \in V \backslash\{s\}$ is fixed, we may consider the subfamily

$$
\mathcal{C}_{s t}=\left\{\delta^{+}(S) \mid s \in S, t \notin S\right\} .
$$

$\mathcal{C}_{s t}$ is closed under the operations $\wedge$ and $\vee$. Moreover, the function $v$ from before remains monotone and supermodular on $\mathcal{C}_{s t}$. It follows that the greedy algorithm now becomes Dijkstra's shortest $(s, t)$-path algorithm, which has been observed already by Johnson [20] (see also [17]).

\subsubsection{Flows in planar graphs}

Let $G=(V, E)$ be an $(s, t)$-planar graph and consider the ordered set $(\mathcal{P}, \preceq)$ of all cycle-free $s, t$-paths as in Ex. 6.2. For any $P, Q \in \mathcal{P}$, set

$$
\begin{aligned}
& P \vee Q=\text { largest path in } G(P \cup Q) \\
& P \wedge Q=\text { lowest path in } G(P \cup Q)
\end{aligned}
$$


(cf. the "switched paths" of Hoffman [16]). Since $P \vee Q$ and $P \wedge Q$ contain only edges from $P \cup Q$, the consecutive order $(\mathcal{P}, \preceq$ ) satisfies the hypothesis of Theorem 6.1 with $v(P)=1$ for all $P \in \mathcal{P}$.

Given capacities $c(e)$ on the edges, the greedy algorithm computes a maximum flow $f \in \mathbb{R}^{E}$ in $G$ as follows: in each iteration, the algorithm sends as much flow as possible along the largest path. More precisely: in each iteration, the algorithm chooses an edge $e$ of minimal capacity $c(e)$ in the largest path $P$ and assigns the flow $f(P)=c(e)$. It reduces the capacities of all edges in $P$ by $c(e)$, removes $e$ from $G$, and continues with the largest path in $G \backslash e$ in the next iteration until finally no $s, t$ path exists anymore. This is the uppermost path algorithm of Ford and Fulkerson [12] (see also, e.g. [1]).

Acknowledgments The authors thank the referees for their comments and remarks on the manuscript, which have helped to improve the presentation of the article.

Open Access This article is distributed under the terms of the Creative Commons Attribution Noncommercial License which permits any noncommercial use, distribution, and reproduction in any medium, provided the original author(s) and source are credited.

\section{References}

1. Borradaile, G., Klein, P.: An $O(n \log n)$ algorithm for maximum $s t$-flow in a directed planar graph. In: SODA, pp. 524-533. ACM, New York. ISBN: 0-89871-605-5 (2006)

2. Choquet, G.: Theory of capacities. Ann. Inst. Fourier 5, 131-295 (1953)

3. Dietrich, B.L., Hoffman, A.J.: On greedy algorithms, partially ordered sets, and submodular functions. IBM J. Res. Dev. 47, 25-30 (2003)

4. Edmonds, J.: Optimum branchings. J. Res. Nat. Bur. Stand. B 71, 233-240 (1967)

5. Edmonds, J.: Submodular functions, matroids and certain polyhedra. In: Proceedings of International Conference on Combinatorics (Calgary) pp. 69-87 (1970)

6. Edelman, P.H., Jamison, R.E.: The theory of convex geometries. Geometriae Dedicata 19, 247-270 (1985)

7. Faigle, U., Fujishige, S.: A general model for matroids and the greedy algorithm. Math. Program. A 119, 353-369 (2009)

8. Faigle, U., Grabisch, M., Heyne, M.: Monge extensions of cooperation and communication structures. Eur. J. Oper. Res. 206, 104-110 (2010)

9. Faigle, U., Kern, W.: On the core of ordered submodular cost games. Math. Program. 87, 483-489 (2000)

10. Faigle, U., Peis, B.: Two-phase greedy algorithms for some classes of combinatorial linear programs. In: Proceedings SODA 2008, San Francisco, pp. 161-166 (2008)

11. Frank, A.: Increasing the rooted-connectivity of a digraph by one. Math. Program. 84, 565-576 (1999)

12. Ford, L.R., Fulkerson, D.R.: Maximal flow through a network. Can. J. Math. 8, 399-404 (1956)

13. Fujishige, S.: Dual greedy polyhedra, choice functions, and abstract convex geometries. Discrete Optim. 1, 41-49 (2004)

14. Fujishige, S.: Submodular Functions and Optimization. 2nd ed. In: Ann. Discrete Mathematics, vol. 58. (2005)

15. Fulkerson, D.R.: Packing rooted directed cuts in a weighted directed graph. Math. Program. 6, 1-13 (1974)

16. Hoffman A.J.: Ordered sets and linear programming. Ordered Sets, pp. 619-654. D. Reidel Publishing Company, Boston (1981)

17. Hoffman, A.J.: On greedy algorithms that succeed. Surveys in Combinatorics. pp. 97-112. Cambridge University Press, Cambridge (1985)

18. Hoffman, A.J., Schwartz, D.E.: On lattice polyhedra. In: Hajnal, A., Sós, V.T. (eds.) Proceedings of 5th Hungarian Conference in Combinatorics, pp. 593-598. North-Holland, Amsterdam (1978) 
19. Hoffman, A.J., Kolen, A.W.J., Sakarowitch, M.: Totally balanced and greedy matrices. SIAM J. Alg. Discr. Methods 6, 721-730 (1985)

20. Johnson, E.: On cut-set integer polyhedra. Cahiers du Centre d'Études de Recherche Opérationnelle 17, 235-251 (1975)

21. Lovász, L. et al.: Submodular functions and convexity. In: Bachem, A. et al. (eds.) Mathematical Programming: The State of the art, pp. 235-257. Springer-Verlag, Berlin (1983)

22. Murota K.: Discrete Convex Analysis. SIAM Monographs on Discrete Mathematics and Applications, vol. 10. Society for Industrial and Applied Mathematics (SIAM), Philadelphia (2003)

23. Moulin, H.: Choice functions over a finite set: a summary. Soc. Choice Welfare 2, 147-160 (1985)

24. Rockafellar, R.T.: Convex Analysis. Princeton University Press, Princeton (1970)

25. Shapley, L.S.: Cores of convex games. Int. J. Game Theory 1, 12-26 (1971) 